\title{
Impedance Analysis of Mobile Phone Antenna in the Presence of User's Hand
}

\author{
Nur Basyirah A Rahman*,A.A.Al-Hadi, and Saidatul Norlyana Azemi \\ School of Computer and Communication Engineering, UniMAP, 02600 Arau, Perlis, Malaysia
}

\begin{abstract}
User interaction (UI) has been identified to degrade the performance of the antenna. A system that measures impedance characteristics of a planar inverted-F antenna (PIFA) when there are user interactions is designed using Keysight VEE Pro. The operational frequencies of the antenna are $900 \mathrm{MHz}$ and $1800 \mathrm{MHz}$ at low band and high band, respectively. The obtained results are compared and then a new impedance matching circuit is proposed and designed by using ADS (Advanced Design System). The proposed matching circuit at each operating frequency is a basic lumped circuit using the L network. Both simulation and experimental work have been done.
\end{abstract}

\section{Introduction}

Mobile phones are now a necessity need for many people throughout the world. Advanced mobile phones are able to receive and place phone calls, store data, take picture and also can be used as walkie-talkie. As the technology advanced, the impact of mobile phones increased accordingly. Therefore, the contact of mobile phones and human body has increased, too. The interaction between users and mobile antenna is considered as very crucial.

It is an immense challenge to ensure that the antenna in mobile terminal meets the crucial criteria. The fundamentals that the antenna should meet are radiation characteristic to ensure the consistency of communication, a wide bandwidth to discourage distortions of transmitted signals, minimal impact on users and minute sensitivity of the antenna parameters to the imminence of users and of course, it should be in small, compact size [1]. Specific Absorption Rate (SAR) is another significant criterion to mull over. SAR measures the amount of power captivated by body tissue when exposed to electromagnetic radiation [2].

Various studies have been done previously that showed the performance of the antenna in mobile terminal are significantly affected by the presence of users, especially when the mobile antenna is being held by the users, close to their heads [1], [3]-[7]. It is produced feeble performance of mobile terminal; the output power and radiation efficiency are worsened by huge mismatch. This mismatch is likely to happen because of the dependency of frequency on the antenna impedance.

Planar inverted-F antenna (PIFA) is a basic antenna that is being used in a wide range of application, especially mobile devices. Besides having simple structure and uncomplicated fabrication, it is also inexpensive to manufacture and inconspicuous, as it is in small volume [8]. The structures of PIFA make it pertinent to be used as a multi-band antenna, hence relevant in mobile devices [2], [9], [10]. The antenna involves is a dual-band PIFA.

This paper is to analyse antenna impedance in free space and also in the presence of human hands and heads, consequently to propose impedance matching circuit to improve the antenna impedance mismatch.

\section{Impedance analysis}

The first stage of the analysis was designing a system to capture the impedance value of the antenna in mobile terminal, and the experiment was conducted when the antenna was in free space and with user interaction (UI). This system was built within a simulation tool, VEE Pro software. The antenna used in this stage is PIFA and the specification will be elaborated more in the next section. The second stage was proposing impedance matching circuit when mismatch occurred in the measurement done in the first phase. This circuit utilized the usage of Advanced Design System (ADS).

\subsection{Software development for impedance measurement}

This stage involved the usage of simulation tool and an antenna prototype, namely Keysight VEE and planar inverted-F antenna (PIFA). These two components worked closely with the network analyzer in this stage of the project. VEE or Visual Engineering Environment is a graphical programming language for building test and measurement application [11].

Keysight VEE was used to implement a system to capture the value of impedance when the antenna was in

* Corresponding author: basyirahrahman@gmail.com 
free space and when human was present. Keysight VEE allows users to alter parameters without reconfiguring programs, thus making it easy to use to measure and record the impedance value at different frequency range, $900 \mathrm{MHz}$ and $1800 \mathrm{MHz}$, respectively.

When the program was executed, it captured the impedance and $\mathrm{S}_{11}(\mathrm{~dB})$ at the specified operating frequencies. The program could only be simulated when the computer was connected to a network analyzer using a GPIB cable or LAN cable.

The PIFA prototype used was a dual-band antenna, fabricated for mobile terminal with 100 (L) x 40(W) $\mathrm{x}$ $0.035(\mathrm{H}) \mathrm{mm} 3$ dimension. The antenna prototype was supplied with $50 \mathrm{ohm}$ coaxial feeding structure. Once the program was successfully simulated without error, the antenna was connected to the network analyzer and the antenna was calibrated to the desired frequency range of $700 \mathrm{MHz}-2000 \mathrm{MHz}$ for easy impedance measurement.

Once the setup was all fixed, the program code was simulated. The first simulation of the program, the antenna was left in free space, as shown in Fig. 1, and without user interaction (UI), as shown if Fig. 2. The next simulation was then carried out with UI and this process was done 20 times. The data were automatically saved in the computer and they could be retrieved to be compared.

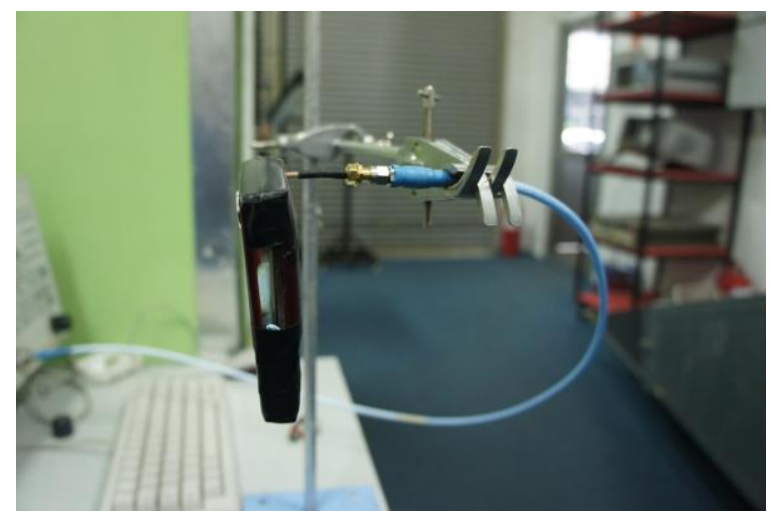

Fig. 1. The antenna in free space

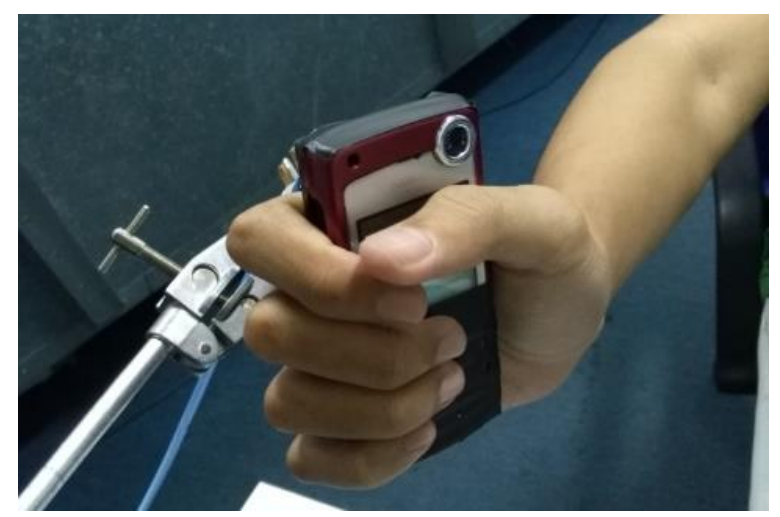

Fig. 2. User gripping the antenna

\subsection{Impedance matching circuit}

In this stage, an impedance matching circuit network was built by using Keysight ADS (Advanced Design System). It provides a built-in design environment [12] and this stage utilizes the workspace to design schematics to display data obtained from network analyzer.

The measurement data were used to compare and contrast the impedance mismatch when user interactions were present. To correct the mismatch, an impedance matching circuit was proposed and designed based on the measurement obtained in the first stage.

The proposal of the impedance matching circuit was made easy by using the feature provided in ADS, the Smith Chart Utility. When the impedance was matched to the desired value, a circuit was automatically designed and the values of the inductor and capacitor, if any, were given.

\section{Results}

The data from the simulation using VEE Pro software for impedance characteristics of antenna with and without user interactions were recorded and analysed, and matching circuits were proposed accordingly.

\subsection{Impedance measurement}

The simulation of the programming in VEE Pro software and the experiments done were focused on the variation of the antenna impedance with user interaction (UI). The data recorded using VEE Pro software is shown in Table 1 and Table 2.

Table 1. Data recorded from VEE Pro software at $900 \mathrm{MHz}$

\begin{tabular}{|c|c|c|}
\hline Experiment & Impedance $(\mathrm{Ohm})$ & $\mathrm{S} 11(\mathrm{db})$ \\
\hline Free Space & $56.20030,-25.70119$ & -12.3 \\
\hline 1 & $7.18044,-9.41670$ & -2.4 \\
\hline 2 & $73.42599,-30.76016$ & -10.3 \\
\hline 3 & $114.01299,-47.13789$ & -6.7 \\
\hline 4 & $107.93769,-82.02252$ & -4.9 \\
\hline 5 & $10.02922,-17.07871$ & -3.1 \\
\hline 6 & $9.02490,-17.28456$ & -2.8 \\
\hline 7 & $10.83449,-15.96367$ & -3.4 \\
\hline 8 & $10.83825,-18.32352$ & -3.3 \\
\hline 9 & $9.04438,-15.85823$ & -2.9 \\
\hline 10 & $108.00266,-45.95788$ & -6.9 \\
\hline 11 & $69.37858,-40.83315$ & -8.9 \\
\hline 12 & $7.76002,-8.78291$ & -2.6 \\
\hline
\end{tabular}




\begin{tabular}{|l|l|l|}
\hline 13 & $157.55675,-48.81924$ & -5.1 \\
\hline 14 & $51.25545,-12.28277$ & -9.1 \\
\hline 15 & $94.54969,-65.05639$ & -6.1 \\
\hline 16 & $75.99115,-52.71152$ & -7.3 \\
\hline 17 & $97.31188,-24.59574$ & -8.9 \\
\hline 18 & $6.85889,-7.77914$ & -2.3 \\
\hline 19 & $8.47236,-5.16774$ & -2.9 \\
\hline 20 & $7.87053,-6.77857$ & -2.7 \\
\hline
\end{tabular}

Table 1 and Table 2 show the values of impedance when the antenna was in free space and with user interactions at $900 \mathrm{MHz}$ and $1800 \mathrm{MHz}$, and the values of $\mathrm{S}_{11}$ in $\mathrm{dB}$. The simulation was first done on the antenna without user interaction. Then the experiments were done with 20 users, recording the impedance and S11 for each user at both frequencies. The measurements were executed using freestyle grips. The antenna was placed in a casing to make the gripping easier. Each user gripped the antenna differently, providing a variation of impedance $(\Omega)$ and $S 11$ values.

Table 2. Data recorded from VEE Pro software at $1800 \mathrm{MHz}$

\begin{tabular}{|c|c|c|}
\hline Experiment & Impedance (Ohm) & $\mathrm{S} 11(\mathrm{db})$ \\
\hline Free Space & $26.79966,33.13157$ & -12.3 \\
\hline 1 & $14.82247,53.02929$ & -2.4 \\
\hline 2 & $28.80263,24.62985$ & -8.1 \\
\hline 3 & $24.65929,13.18249$ & -8.5 \\
\hline 4 & $22.76062,23.49544$ & -6.5 \\
\hline 5 & $24.09021,49.19400$ & -4.1 \\
\hline 6 & $26.35174,45.86049$ & -4.7 \\
\hline 7 & $23.59299,46.91540$ & -4.2 \\
\hline 8 & $20.62890,46.00855$ & -3.8 \\
\hline 9 & $22.34369,52.98932$ & -3.5 \\
\hline 10 & $18.69014,22.75773$ & -5.4 \\
\hline 11 & $30.35454,24.68291$ & -8.5 \\
\hline 12 & $13.14519,49.63192$ & -2.3 \\
\hline 13 & $23.93606,13.63711$ & -8.2 \\
\hline 14 & $27.97118,26.95319$ & -7.4 \\
\hline 15 & $28.57141,25.01479$ & -7.9 \\
\hline 16 & $29.50213,21.57662$ & -8.9 \\
\hline 17 & $36.77092,26.56201$ & -9.7 \\
\hline
\end{tabular}

\begin{tabular}{|l|l|l|}
\hline 18 & $14.69248,58.97473$ & -2.1 \\
\hline 19 & $18.86003,62.04056$ & -2.5 \\
\hline 20 & $18.62644,63.33371$ & -2.4 \\
\hline
\end{tabular}

Mobile antenna is usually designed to operate ideally in free space. These experiments proved that having user interactions cause impedance mismatch that varies with frequencies at high band and low band. Human body is made of tissues that are highly likely of having high permittivity that causes the detuning of antenna. Each user also has different hand size and different strength in gripping the antenna that absorbs different amount of power, leading to the decrease of efficiency.

\subsection{S-Parameters and smith charts}

The variation of S-parameters and the smith chart for impedance variation at low band and high band are shown in Fig. 3, Fig. 4, Fig. 5 and Fig. 6. The single black line iss the measurement made in free space, and the coloured lines are the measurement made on 20 different users. Based on the smith chart, it can be seen that user interactions have caused several severe impedance mismatch.

It is duly noted that the impedance at $900 \mathrm{MHz}$ and $1800 \mathrm{MHz}$ measured in free space were not properly matched. There are several circumstances that can be considered as the result of the impedance mismatch in free space. One of the factors is the fabrication tolerance. There might have been a minor miscalculation in the antenna size or a slight error during fabrication. A loose antenna port and the material used as the antenna casing should be taken into consideration too. The size of the antenna must be accurate and the port must be properly intact for the antenna to operate at high performance in free space.

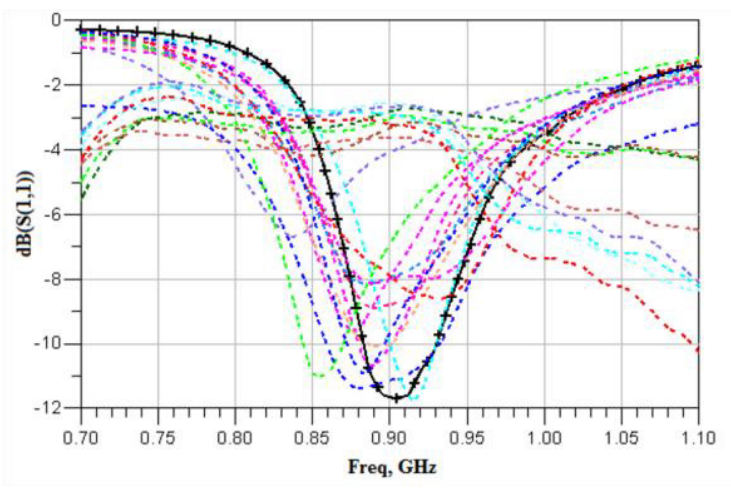

Fig. 3. Frequency $(\mathrm{GHz})$ against $\mathrm{S} 11(\mathrm{~dB})$ of antenna at low band 


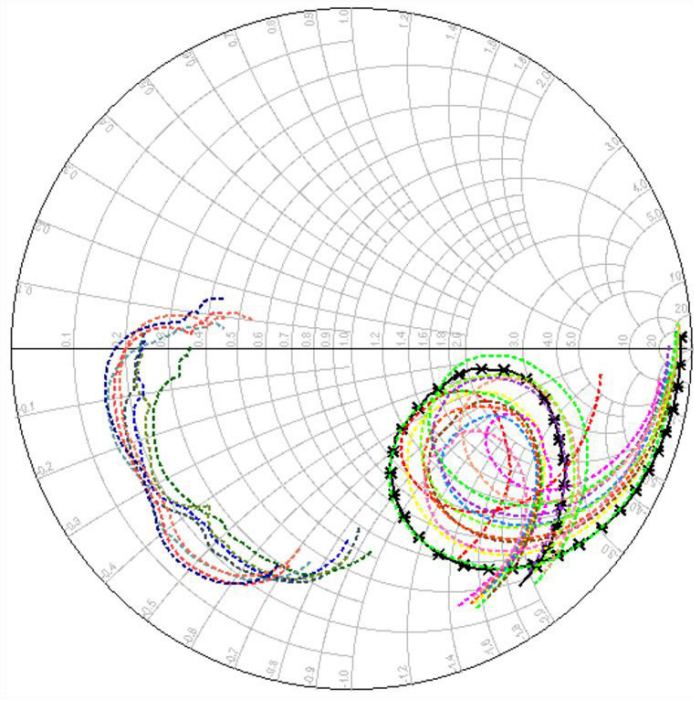

Fig. 4. Smith chart for impedance variation at low band

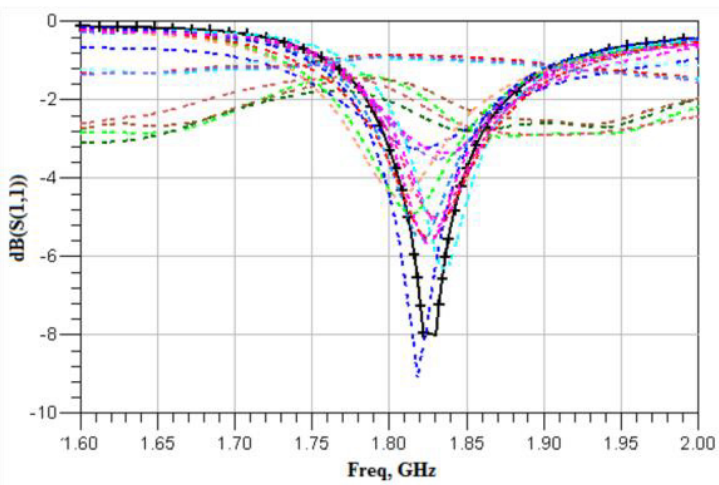

Fig. 5. Frequency $(\mathrm{GHz})$ against $\mathrm{S} 11(\mathrm{~dB})$ of antenna at high band

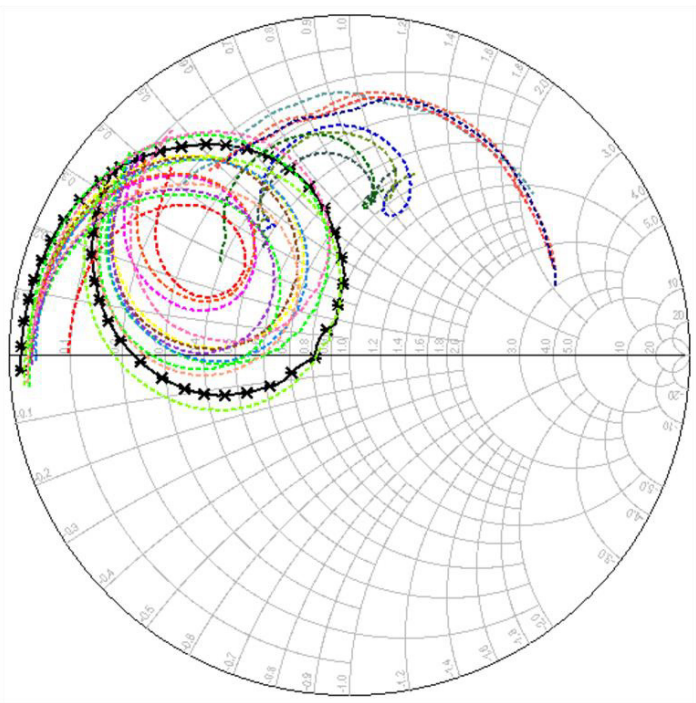

Fig. 6. Smith chart for impedance variation at high band

\subsection{Impedance matching circuit}

Fig. 7 shows that the impedance mismatch at $900 \mathrm{MHz}$ is more likely to happen in the $51.25 \Omega-157.55 \Omega$ impedance range. An average impedance value of $91.42 \Omega$ is selected.

The average $\mathrm{Z}$ value of 91.4203 - j43.2616 $\Omega$ is inserted in the space provided and then a matching circuit consists of an inductor (L) and a capacitor (C) is designed, as shown in Fig. 8. The values of the inductor and capacitor used in the circuit are $10.0299 \mathrm{nH}$ and $1.0003 \mathrm{pF}$ respectively, as shown in Fig. 9.

Fig. 10 shows that the impedance mismatch at $1800 \mathrm{MHz}$ is more likely to happen in the $13.15 \Omega-$ $36.78 \Omega$ impedance range. An average impedance value of $23.62 \Omega$ is selected.

The average $Z$ value of $23.6178+j 37.3144 \Omega$ is inserted in the space provided. Another matching circuit consists of two capacitors (C) is designed, as shown in Fig. 11. The value of $\mathrm{C} 1$ is $2.2502 \mathrm{pF}$ and the value of $\mathrm{C} 2$ is $0.8317 \mathrm{pF}$, as shown in Fig. 12 .

The results from the experiments done on the effect of user interactions (UI) on mobile antenna and the possible causes of antenna when the antenna is being held by user have been analyzed and discussed. Matching circuits for mismatched impedance at low band and high band have also been designed. This study has successfully achieved its objectives to design a system that measure the impedance of antenna in mobile antenna, to study the behavior of impedance in free space and in the presence of user interaction and to propose impedance matching circuit for each operational frequency.

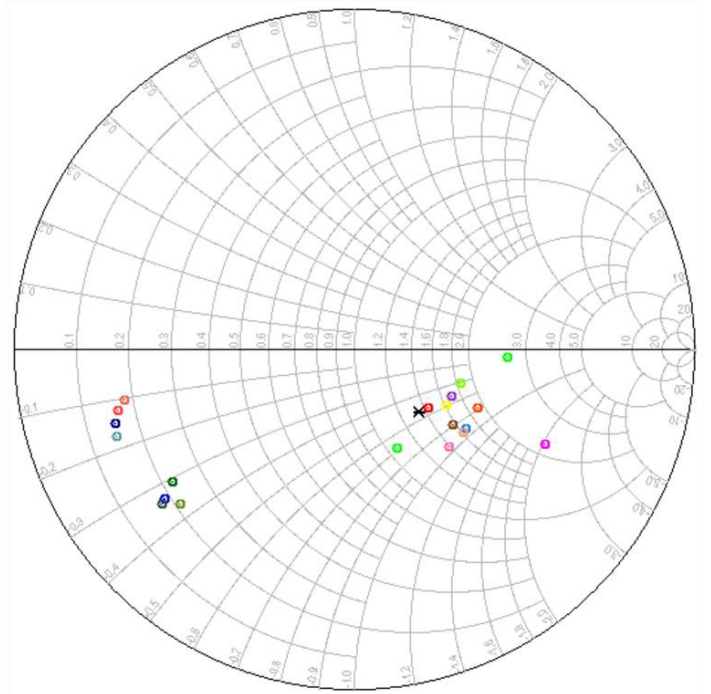

Fig. 7. Impedance variation at $900 \mathrm{MHz}$ 


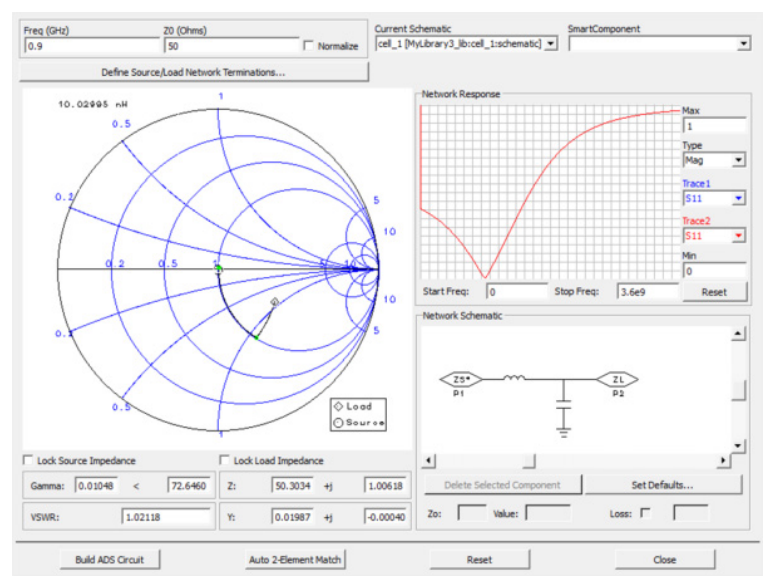

Fig. 8. Designing matching circuit at $900 \mathrm{MHz}$

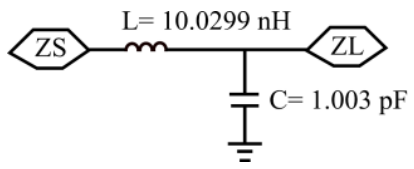

Fig. 9. Impedance matching circuit $900 \mathrm{MHz}$

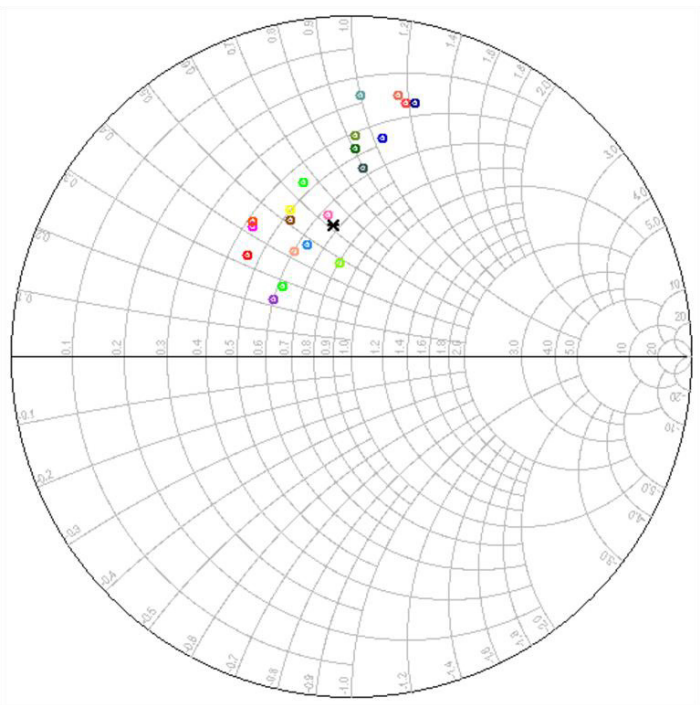

Fig. 10. Impedance variation at $1800 \mathrm{MHz}$

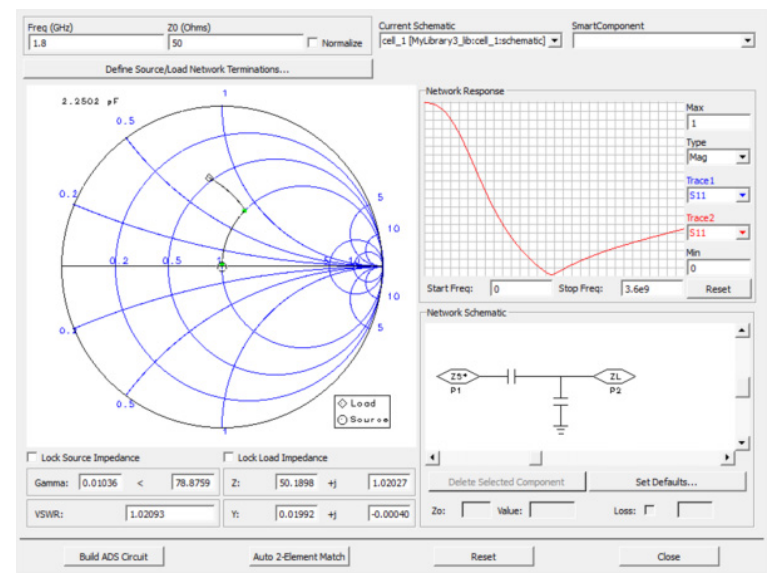

Fig. 11. Designing matching circuit at $1800 \mathrm{MHz}$

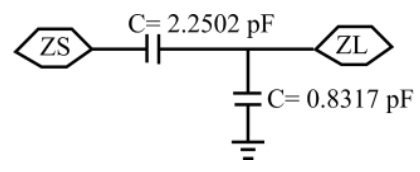

Fig. 12. Impedance matching circuit at $1800 \mathrm{MHz}$

\section{Conclusion}

The variation of impedance and $\mathrm{S}_{11}$ measurement caused by user interactions were because each user had different method of holding or gripping the antenna. The size of user's hand and the strength of their grips also affected the performance of antenna, in some cases, very severely. The absorbed power by each user's hand was different too.

The measurements of impedance obtained from the experiments were then used to propose matching circuits for impedance mismatch at each band; low band and high band. These matching circuits are highly important in tuning mismatched impedance at mobile antenna terminal. They help to keep the antenna to be in good performance even when there is user interaction.

The proposed matching circuit in this study, a simple lumped circuit using the L network, fully utilized the Smith Chart Utility feature on ADS. The circuit designed at low band is made up of an inductor and a capacitor, while at high band, the circuit designed is made up of two capacitors of different values.

The authors would like to thank the Ministry of Science, Technology and Innovation for the financial support under eSciencefund (Reference Code: 01-01-015-SE0258)

\section{References}

1. R. Przesmycki, M. Bugaj, L. Nowosielski, M. Wnuk, Dual-band mobile phone antenna for RF protection of human head, 2011 SBMO/IEEEMTT-S Int. Microw. Optoelectron. Conf. (IMOC 2011), 235-238 (2011)

2. Z. Ying, Antennas in cellular phones for mobile communications, Proc. IEEE, 100, 2286-2296 (2012)

3. K. Boyle, M. Leitner, Mobile phone antenna impedance variations with real users and phantoms, Final Progr. B. Abstr. - iWAT 20112011 IEEE Int. Work. Antenna Technol. Small Antennas, Nov. Struct. Innov. Metamaterials, 1, 420-423 (2011)

4. K. Zhao, S. Zhang, Z. Ying, T. Bolin, S. He, SAR Study of different MIMO antenna designs for LTE application in smart mobile Phones, 2-3 (2012)

5. M. Lin, C. Chiu, Human-body effects on the design of card-type UHF RFID tag antennas (2011)

6. Q. Guo, R. Mittra, F. Lei, Z. Li, J. Ju, J. Byun, Interaction between internal antenna and external antenna of mobile phone and hand effect, Antennas Propagation, IEEE Trans., 61, 862-870 (2013)

7. K. R. Boyle, Y. Yuan, L. P. Ligthart, Analysis of mobile phone antenna impedance variations with 
user proximity, IEEE Trans. Antennas Propag., 55, 364-372 (2007)

8. N. A. Saidatul, P. J. Soh, Y. Sun, D. Lauder, and A. A. H. Azremi, Multiband fractal PIFA (Planar Inverted $\mathrm{F}$ Antenna) for mobile phones, 671-675 (2010)

9. P. Tsai, D. Lin, H. Lin, and P. Chen, Printed monopole slot antenna for internal multiband mobile phone antenna, 55, 3690-3697 (2007)

10. C. Rowell and E. Y. Lam, Mobile-phone antenna design, IEEE Antennas Propag. Mag., 54, 14-34 (2012)

11. Agilent Technologies, Agilent VEE Pro VEE Pro Users Guide (2014)

12. Keysight Technologies, Keysight Technologies EDA Advanced Design System (2014) 